\title{
Serum interleukin-16 significantly correlates with the Vasculitis Damage Index in antineutrophil cytoplasmic antibody- associated vasculitis
}

Taejun Yoon ${ }^{1 \dagger}$, Jung Yoon $\mathrm{Pyo}^{2 \dagger}$, Sung Soo Ahn², Jason Jungsik Song ${ }^{2,3}$, Yong-Beom Park ${ }^{2,3}$ and Sang-Won Lee ${ }^{2,3^{*}}$ (D)

\begin{abstract}
Background: Interleukin (IL)-16 is a T cell chemoattractant produced by peripheral mononuclear cells. We investigated whether IL-16 plays a pro- or an anti-inflammatory role in antineutrophil cytoplasmic antibodyassociated vasculitis (AAV). Furthermore, we investigated whether the level of IL-16 could predict the activity and extent of organ damage in AAV based on AAV-specific indices.

Methods: Seventy-eight patients with AAV from a prospective observational cohort were included in this analysis. Blood sampling and clinical assessments, including the Birmingham Vasculitis Activity Score (BVAS), Five-Factor Score (FFS), Short Form 36-item Health Survey (SF-36), and Vasculitis Damage Index (VDI), were performed, and laboratory data were collected. Serum IL-16 was measured from stored sera.

Results: The median age was 62.0 years, and 27 patients were male. The median serum IL-16 concentration was $84.1 \mathrm{pg} / \mathrm{dL}$, and the median BVAS, FFS, VDI, and SF-36 scores were 7.0, 1.0, 3.0, and 48.0, respectively. Among the AAV-related indices, the serum IL-16 concentration was correlated with VDI $\left(R^{2}=0.306, P=0.006\right)$, but not with $\operatorname{BVAS}\left(R^{2}=0.024, P=0.834\right)$, FFS $\left(R^{2}=-0.069, P=0.550\right)$, or SF-36 $\left(R^{2}=-0.015, P=0.898\right)$. The serum IL-16 concentration also did not correlate with either the erythrocyte sedimentation rate or the C-reactive protein concentration. Per our analysis based on organ involvement, only patients with ear, nose, and throat manifestations had higher serum IL-16 concentrations relative to those with other conditions $(P=0.030)$.

Conclusions: This was the first study to elucidate the clinical implication of serum IL-16 in patients with AAV. We found that the serum IL-16 level may reflect the cross-sectional VDI scores among AAV-specific indices. Future studies with larger numbers of patients and serial measurements could provide more reliable data on the clinical implications of serum IL-16 in AAV.
\end{abstract}

Keywords: Antineutrophil cytoplasmic antibody-associated vasculitis, Serum IL-16, Activity, Vasculitis damage, Reflect

\footnotetext{
* Correspondence: sangwonlee@yuhs.ac

${ }^{\dagger}$ Taejun Yoon and Jung Yoon Pyo contributed equally to this work and are co-first authors

${ }^{2}$ Division of Rheumatology, Department of Internal Medicine, College of Medicine, Yonsei University, 50-1 Yonsei-ro, Seodaemun-gu, Seoul 03722, Republic of Korea

${ }^{3}$ Institute for Immunology and Immunological Diseases, College of Medicine, Yonsei University, Seoul, Republic of Korea

Full list of author information is available at the end of the article
}

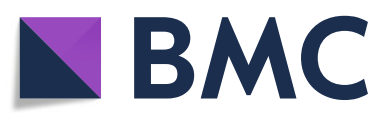

() The Author(s). 2020 Open Access This article is licensed under a Creative Commons Attribution 4.0 International License, which permits use, sharing, adaptation, distribution and reproduction in any medium or format, as long as you give appropriate credit to the original author(s) and the source, provide a link to the Creative Commons licence, and indicate if changes were made. The images or other third party material in this article are included in the article's Creative Commons licence, unless indicated otherwise in a credit line to the material. If material is not included in the article's Creative Commons licence and your intended use is not permitted by statutory regulation or exceeds the permitted use, you will need to obtain permission directly from the copyright holder. To view a copy of this licence, visit http://creativecommons.org/licenses/by/4.0/. The Creative Commons Public Domain Dedication waiver (http://creativecommons.org/publicdomain/zero/1.0/) applies to the data made available in this article, unless otherwise stated in a credit line to the data. 


\section{Background}

Antineutrophil cytoplasmic antibody (ANCA)-associated vasculitis (AAV) refers to a group of systemic vasculitides that affect small vessels, including microscopic polyangiitis (MPA), granulomatosis with polyangiitis (GPA), and eosinophilic granulomatosis with polyangiitis (EGPA) [1, 2]. In clinical practice, the following are often assessed at every visit: Birmingham Vasculitis Activity Score (BVAS), version 3 for activity; Five-Factor Score (FFS) for prognosis; the Korean version of the Short Form 36-item Health Survey (SF-36) for functional status; and the Vasculitis Damage Index (VDI) for end organ damage [3-6]. Although a single serum biomarker cannot replace each AAV-specific index, such a marker may have clinical implications as an immediate and convenient indicator if it reflects a specific AAV-related index. Therefore, the identification of a serologic biomarker that can estimate a cross-section of the many AAV-specific indices would be useful.

Interleukin (IL)-16 was first introduced as a $\mathrm{T}$ cell chemoattractant produced by peripheral mononuclear cells in 1982 [7]. This cytokine is produced by various cells, such as CD4+ T cells, CD8+ T cells, mast cells, eosinophils, and fibroblasts [8]. Bioactive IL-16 induces the chemotaxis of $\mathrm{CD} 4+\mathrm{TH} 1$ cells by binding to CD4 to enhance $\mathrm{T}$ cell proliferation and stimulate $\mathrm{B}$ cell development, while inhibiting antigen-triggered TH2 cellmediated inflammation and impairing macrophage inflammatory protein (MIP)-1beta and stromal cell-derived factor (SDF)-1alpha-mediated T cell chemoattraction [9]. Previous reports have highlighted the clinical significance of serum IL-16 in several typical systemic autoimmune diseases.

Serum IL-16 was observed to be significantly elevated in patients with systemic lupus erythematosus (SLE) relative to healthy controls, and the level of this cytokine was correlated with SLE activity [10]. Unlike SLE, the clinical role of serum IL-16 remains controversial in rheumatoid arthritis (RA). It was suggested that IL-16 production may be accelerated by IL-17 in RA and that serum IL-16 correlated with disease activity reflecting pro-inflammatory effect $[11,12]$. However, there was a report that treatment with exogenous IL-16 inhibits the production of pro-inflammatory cytokines in rheumatoid synovitis, demonstrating the anti-inflammatory effects of IL-16 [13].

Because SLE, RA, and AAV share inflammatory mechanisms, we aimed to investigate whether serum IL-16 plays a pro- or an anti-inflammatory role in AAV. Furthermore, we wondered whether serum IL-16 might reflect the inflammatory burden of small pro-inflammatory molecules or reflect a consequence of extensive tissue damage. However, no previous study has elucidated the clinical implication of serum IL-16 in patients with AAV. In this study, we measured the serum IL-16 levels in patients with AAV and investigated the association of this factor with the cross-sectional scores of AAV-specific indices, such as the BVAS, FFS, SF-36, and VDI.

\section{Methods \\ Patients}

We included 78 patients with AAV in this study. The patients were enrolled in the Severance Hospital ANCAassociated VasculitidEs (SHAVE) cohort, a prospective observational cohort of patients with MPA, GPA, and EGPA, from November 2016 to May 2019. All patients were newly diagnosed with AAV at the Division of Rheumatology, Department of Internal Medicine, Yonsei University College of Medicine and Severance Hospital. The patients met the criteria of the 2007 European Medicine Agency algorithms for AAV and polyarteritis nodosa (the 2007 EMA algorithm) and the 2012 revised International Chapel Hill Consensus Conference Nomenclature of Vasculitides $[1,2]$. At the time of diagnosis, patients with serious infections, including hepatitis $B$ or $\mathrm{C}$ virus infection, malignancies, or secondary vasculitis features related to autoimmune diseases, were excluded from the SHAVE cohort. In addition, all patients were immunosuppressant-naïve at the enrollment of this study. This study was approved by the Institutional Review Board of Severance Hospital (4-2016-0901), and written informed consent was obtained from the patients at the time of blood sampling.

\section{Clinical and laboratory data}

The collected demographic data included age and sex. The clinical manifestations were counted based on the items in BVAS version 3. We obtained the following laboratory data at diagnosis: perinuclear (P)-ANCA, cytoplasmic (C)-ANCA, myeloperoxidase (MPO)-ANCA, proteinase 3 (PR3)-ANCA, and acute-phase reactants, including erythrocyte sedimentation rate (ESR) and Creactive protein (CRP). We also collected AAV-specific indices such as the BVAS version 3, FFS, SF-36, and VDI scores on the same day as the blood sampling and clinical assessment. According to the SHAVE cohort protocol, we obtained whole blood from each consenting patient, immediately isolated sera, and stored the samples at $-80^{\circ} \mathrm{C}$. Serum IL-16 concentrations in stored sera were measured using ELISA kits (Abcam, Cambridge, UK) according to the manufacturer's instructions.

\section{Statistical analyses}

All statistical analyses were conducted using SPSS software (version 25 for Windows; IBM Corp., Armonk, NY, USA). Continuous variables are expressed as means with standard deviations, and categorical variables are expressed as numbers (percentages). The correlation coefficient between the two variables was obtained using a 
Spearman correlation analysis. Significant differences in continuous variables between the two groups were compared using the Mann-Whitney test. $P$ values $<0.05$ were considered statistically significant.

\section{Results}

\section{Characteristics of AAV patients}

The baseline characteristics of the patients are described in Table 1. The median age was 62.0 years, and $27 \mathrm{pa}$ tients were men. The median BVAS, FFS, and VDI scores were 7.0, 1.0, and 3.0, respectively.

MPO-ANCA and PR3-ANCA were detected in 43 and 7 patients, respectively. The most common clinical manifestation was pulmonary (64.1\%), followed by renal and ear, nose, and throat (ENT) manifestations. The median ESR and CRP levels were $37.5 \mathrm{~mm} / \mathrm{h}$ and $2.6 \mathrm{mg} / \mathrm{L}$, respectively. The median serum IL-16 level was $84.1 \mathrm{pg} /$ $\mathrm{mL}$.

\section{Correlation analysis}

Of the AAV-specific indices, the serum IL-16 concentration correlated with the cross-sectional VDI scores $\left(R^{2}=\right.$ $0.306, P=0.006)$, but not with the BVAS, FFS, or SF-36 scores or with ESR or CRP (Table 2).

\section{Comparative analysis}

We compared the serum IL-16 concentration according to each organ or system wherein the involvement frequency exceeded 25\%. Among the general, ENT, pulmonary, and renal manifestations, only patients with ENT manifestations exhibited a higher serum IL-16 concentration than those without ENT manifestations $(P=$ 0.030 ; Table 3 ). In a correlation analysis of the serum IL-16 concentration and the total weighted score per each organ or system involvement, the former was correlated with only the total weighted score of ENT manifestations $\left(R^{2}=0.279, P=0.013\right)$.

Also, we compared the serum IL-16 concentration according to the ANCA status and specific disease type. IL-16 was significantly higher in patients with PR3ANCA than those with MPO-ANCA or those without ANCA $(P<0.001$; Table 4$)$. IL-16 was higher in patients with GPA than those with MPA or EGPA although statistically not significant.

\section{Discussion}

In this study, we demonstrated a correlation of the serum IL-16 concentration with cross-sectional VDI scores, but not with other AAV-specific indices. In addition, the serum IL-16 concentration differed significantly between patients with ENT manifestations and those without.

This study has raised many questions. For example, why does the serum IL-16 level correlate with only the
Table 1 Characteristics of the clinical and laboratory data of 78 patients with AAV

\begin{tabular}{|c|c|}
\hline Variables & Values \\
\hline \multicolumn{2}{|l|}{ Demographic data } \\
\hline Age (years) & $62.0(21.0)$ \\
\hline Male sex (N (\%)) & $27(34.6)$ \\
\hline \multicolumn{2}{|l|}{ Variants $(N(\%))$} \\
\hline MPA & $40(51.3)$ \\
\hline GPA & $21(26.9)$ \\
\hline EGPA & $17(21.8)$ \\
\hline \multicolumn{2}{|l|}{ AAV-specific indices } \\
\hline BVAS & $7.0(11.0)$ \\
\hline FFS & $1.0(1.0)$ \\
\hline VDI & $3.0(2.0)$ \\
\hline SF-36 PCS score & $48.0(36.2)$ \\
\hline SF-36 MCS score & $57.0(34.5)$ \\
\hline \multicolumn{2}{|l|}{ ANCA positivity (N (\%)) } \\
\hline MPO-ANCA positivity & $43(55.1)$ \\
\hline PR3-ANCA positivity & $7(9.0)$ \\
\hline ANCA negativity & $28(35.9)$ \\
\hline \multicolumn{2}{|l|}{ Clinical manifestations $(N(\%))$} \\
\hline General & $27(34.6)$ \\
\hline Cutaneous & $9(11.5)$ \\
\hline Mucous membrane and eye & $5(6.4)$ \\
\hline Ear, nose, and throat & $36(46.2)$ \\
\hline Pulmonary & $50(64.1)$ \\
\hline Cardiovascular & $5(6.4)$ \\
\hline Abdominal & $0(0)$ \\
\hline Renal & $39(50.0)$ \\
\hline Nervous system & $17(21.8)$ \\
\hline \multicolumn{2}{|l|}{ Acute-phase reactants } \\
\hline ESR $(\mathrm{mm} / \mathrm{h})$ & $37.5(44.0)$ \\
\hline CRP (mg/L) & $2.6(14.6)$ \\
\hline IL-16 (pg/mL) & $84.1(198.7)$ \\
\hline
\end{tabular}

Values are expressed as medians (interquartile ranges, IQR) or numbers (percentages)

AAV ANCA-associated vasculitis, ANCA antineutrophil cytoplasmic antibody, MPA microscopic polyangiitis, GPA granulomatosis with polyangiitis, EGPA eosinophilic granulomatosis with polyangiitis, BVAS Birmingham Vasculitis Activity Score, FFS Five-Factor Score, VDI Vasculitis Damage Index, SF-36 Short Form-36, $P$ perinuclear, $C$ cytoplasmic, MPO myeloperoxidase, $P R-3$ proteinase 3, AST aspartate aminotransferase, ALT alanine aminotransferase, ESR erythrocyte sedimentation rate, $C R P C$-reactive protein

cross-sectional VDI scores? The first hypothesis is that serum IL-16 might be associated with fibrotic changes. Compared to BVAS, FFS, or acute-phase reactants, the VDI of AAV may be more strongly associated with chronic inflammation (late phase or fibrotic phase) than with acute inflammation (early phase or pro-fibrotic phase) [14]. Kazuo et al. reported that IL-16 levels 
Table 2 Correlation analysis of serum IL-16 with continuous variables in 78 patients with AAV

\begin{tabular}{lll}
\hline Variables & Correlation coefficient $\left(\boldsymbol{r}^{2}\right)$ & $P$ value \\
\hline AAV-specific indices & & \\
BVAS & 0.024 & 0.834 \\
FFS & -0.069 & 0.550 \\
VDI & 0.306 & 0.006 \\
SF-36 PCS score & -0.015 & 0.898 \\
SF-36 MCS score & -0.122 & 0.288 \\
Acute phase reactants & & \\
ESR (mm/h) & -0.034 & 0.769 \\
CRP (mg/L) & -0.007 & 0.952 \\
\hline
\end{tabular}

AAV ANCA-associated vasculitis, ANCA antineutrophil cytoplasmic antibody, BVAS Birmingham Vasculitis Activity Score, FFS Five-Factor Score, VDI Vasculitis Damage Index, SF-36 Short Form-36, AST aspartate aminotransferase, ALT alanine aminotransferase, ESR erythrocyte sedimentation rate, CRP C-reactive protein

correlated with skin involvement in patients with systemic sclerosis reflecting the role of IL-16 in skin fibrosis [15]. Furthermore, there was a study showing that profibrotic mediators induce IL-16 upregulation and IL-16 gene expression was elevated in lung tissue from patient with idiopathic pulmonary fibrosis [16]. Therefore, we assume that the positive correlation between serum IL16 and VDI may reflect the extent of end organ damage due to progressive fibrosis.

The second hypothesis is that serum IL-16 might be associated with the secondary necrosis of neutrophils. Considering the importance of neutrophil in pathogenesis of AAV, we focused on the relation between IL-16 and neutrophil. It was previously revealed that in neutrophils, IL-16 was stored in a preformed form and that it was passively released during secondary necrosis rather than being actively released from viable or apoptotic cells [17]. Under normal circumstances, neutrophils have a short lifespan and are cleared via apoptosis once their function has ended. However, apoptotic neutrophils may

Table 3 Mean serum IL-16 levels based on organ or system involvement (frequency $\geq 25 \%$ ) in AAV

\begin{tabular}{llll}
\hline Involved organ system & Absence & Presence & $P$ value \\
\hline General & $167.4 \pm 188.5$ & $124.2 \pm 137.6$ & 0.297 \\
Ear, nose, and throat & $112.3 \pm 142.5$ & $199.2 \pm 194.5$ & 0.030 \\
Pulmonary & $147.0 \pm 130.3$ & $155.4 \pm 194.0$ & 0.839 \\
Renal & $151.0 \pm 151.9$ & $153.8 \pm 193.7$ & 0.945 \\
\hline
\end{tabular}

Values are expressed as medians (interquartile ranges, IQR) or numbers (percentages)

AAV ANCA-associated vasculitis, ANCA antineutrophil cytoplasmic antibody, MPA microscopic polyangiitis, GPA granulomatosis with polyangiitis, EGPA eosinophilic granulomatosis with polyangiitis, BVAS Birmingham Vasculitis Activity Score, FFS Five-Factor Score, VDI Vasculitis Damage Index, SF-36 Short Form-36, $P$ perinuclear, $C$ cytoplasmic, MPO myeloperoxidase, $P R-3$ proteinase 3, AST aspartate aminotransferase, $A L T$ alanine aminotransferase, ESR erythrocyte sedimentation rate, CRP C-reactive protein
Table 4 Mean serum IL-16 levels based on disease type and ANCA status

\begin{tabular}{llc}
\hline Variables & Serum IL-16 & $P$ value \\
\hline Disease type & & \\
MPA & $128.3 \pm 151.2$ & 0.162 \\
GPA & $213.9 \pm 224.2$ & \\
EGPA & $133.1 \pm 136.0$ & \\
ANCA status & & $<0.001$ \\
MPO-ANCA positivity & $133.1 \pm 143.3$ & \\
PR3-ANCA positivity & $408.1 \pm 275.0$ & \\
ANCA negativity & $133.1 \pm 143.3$ & \\
\hline
\end{tabular}

ANCA antineutrophil cytoplasmic antibody, MPA microscopic polyangiitis, GPA granulomatosis with polyangiitis, EGPA eosinophilic granulomatosis with polyangiitis, MPO myeloperoxidase, $P R 3$ proteinase 3

not be properly cleared in chronic inflammatory situations, and secondary necrosis may occur. We assumed that in late-phase AAV, accumulated neutrophils undergo secondary necrosis and secrete IL-16, which causes tissue damage. This process may explain the positive correlation between serum IL-16 and VDI of AAV.

The association between ENT involvement and serum IL-16 may be secondary to the phase difference between acute inflammation (early phase or pro-fibrotic phase) and chronic inflammation (late phase or fibrotic phase). We therefore analyzed the sub-items of four frequent manifestations per phase. Regarding the general manifestations, all sub-items such as myalgia, arthralgia, fever, and weight loss may be closely related to the acute phase. Regarding the pulmonary manifestations, five of seven sub-items may be somewhat related to the acute phase. Likewise, regarding the renal manifestations, four of five sub-items may also be related to acute inflammation or exacerbation. Conversely, with respect to ENT manifestations, four of five sub-items, such as paranasal sinusitis (thickened sinus wall), subglottic stenosis, conductive hearing loss, and sensorineural hearing loss, tend to be related to the relatively chronic phase of AAV [3].

However, there is a possibility that the degree of inflammatory burden of renal and lung involvement in our patient population was not very high. Despite $50.0 \%$ and $64.1 \%$ of our patients had renal and pulmonary involvement, our patient's median BVAS was 7.0, implying that the inflammation of renal and pulmonary manifestation was not highly active. This could partly be the factor that serum IL-16 correlating only with ENT involvement. Further research with larger population is needed to clarify the relationship between serum-IL-16 and organ involvement.

Another hypothesis regarding the association of serum-IL16 with ENT involvement is that IL-16 might have an association with PR3-ANCA. PR3-ANCA tends to have dominant ENT involvement and is associated 
more with the GPA than MPA or EGPA. Serum IL-16 in our study was significantly higher in patients with PR3ANCA. One possible explanation for this phenomenon is that IL-16 could play a role in causing granuloma. There was a report showing that high levels of IL-16 were seen in immunohistochemical staining of sarcoidosis granuloma [18]. Regarding that the PR3-ANCA is more relevant in GPA than in MPA, there is a possibility of IL-16 being associated with granuloma. However, further research is needed to understand the relationship between serum IL16 and PR3-ANCA.

One strength of this study is that it is the first to demonstrate an association of serum IL-16 with crosssectional VDI scores, but not with BVAS or FFS scores or acute -phase reactants. Furthermore, we excluded patients with other underlying serious medical conditions, and IL-16 was measured from the stored sera which was drawn before starting immunosuppressant drugs, which can minimize the confounding factors. However, our study also has several limitations. First, the sample size was not large enough to ensure that the findings are generalizable to all patients with AAV. Second, we cannot provide serial or paired results, despite the inclusion of patients with AAV in the prospective cohort. Third, we could not assess the duration from symptom to diagnosis, which might be informative to evaluate the acute or chronic status due to the complexity of the disease.

\section{Conclusions}

In conclusion, the serum IL-16 level may reflect the cross-sectional VDI scores among AAV-specific indices. Future studies with larger numbers of patients and serial measurements could provide more reliable data on the clinical implications of serum IL-16 in AAV.

\section{Abbreviations \\ AAV: Antibody-associated vasculitis; ANCA: Antineutrophil cytoplasmic antibody; BVAS: Birmingham Vasculitis Activity Score; CRP: C-reactive protein; EGPA: Eosinophilic granulomatosis with polyangiitis; ENT: Ear, nose, and throat; ESR: Erythrocyte sedimentation rate; FFS: Five-Factor Score; FVSG: French Vasculitis Study Group; GPA: Granulomatosis with polyangiitis; MPA: Microscopic polyangiitis; RA: Rheumatoid arthritis; SHAVE: Severance Hospital ANCA-associated VasculitidEs; SLE: Systemic lupus erythematosus; VDI: Vasculitis Damage Index}

\section{Acknowledgements}

None.

\section{Authors' contributions}

All authors contributed to data analyses and data interpretation. TY and SWL performed the experiments. SWL also contributed in the study design, statistics, and manuscript preparation. JYP involved in data interpretation and manuscript preparation. SSA, JJS, and YBP participated in the study design and interpretation of the results. The authors read and approved the final manuscript.

\section{Funding}

This research was supported by the Basic Science Research Program through the National Research Foundation of Korea (NRF), funded by the Ministry of Education (2017R1D1A1B03029050), and by a grant from the Korea Health
Technology R\&D Project through the Korea Health Industry Development Institute, funded by the Ministry of Health and Welfare, Republic of Korea (HI14C1324).

\section{Availability of data and materials}

The datasets used and/or analyzed during the current study are available from the corresponding author on reasonable request.

\section{Ethics approval and consent to participate}

This study was approved by the Institutional Review Board of Severance Hospital (4-2016-0901), and written informed consent was obtained from the patients at the time of blood sampling.

Consent for publication

Not applicable.

\section{Competing interests}

The authors declare that they have no competing interests.

\section{Author details}

'Department of Medical Science, BK21 Plus Project, College of Medicine, Yonsei University, Seoul, Republic of Korea. ${ }^{2}$ Division of Rheumatology, Department of Internal Medicine, College of Medicine, Yonsei University, 50-1 Yonsei-ro, Seodaemun-gu, Seoul 03722, Republic of Korea. ${ }^{3}$ Institute for Immunology and Immunological Diseases, College of Medicine, Yonsei University, Seoul, Republic of Korea.

Received: 7 February 2020 Accepted: 30 March 2020

Published online: 07 April 2020

\section{References}

1. Jennette JC, Falk RJ, Bacon PA, Basu N, Cid MC, Ferrario F, et al. 2012 revised International Chapel Hill Consensus Conference Nomenclature of Vasculitides. Arthritis Rheum. 2013:65:1-11.

2. Watts R, Lane S, Hanslik T, Hauser T, Hellmich B, Koldingsnes W, et al. Development and validation of a consensus methodology for the classification of the ANCA-associated vasculitides and polyarteritis nodosa for epidemiological studies. Ann Rheum Dis. 2007:66:222-7.

3. Mukhtyar C, Lee R, Brown D, Carruthers D, Dasgupta B, Dubey S, et al. Modification and validation of the Birmingham Vasculitis Activity Score (version 3). Ann Rheum Dis. 2009:68:1827-32.

4. Guillevin L, Pagnoux C, Seror R, Mahr A, Mouthon L, Le Toumelin P, et al. The Five-Factor Score revisited: assessment of prognoses of systemic necrotizing vasculitides based on the French Vasculitis Study Group (FVSG) cohort. Medicine (Baltimore). 2011;90:19-27.

5. Bhamra K, Luqmani R. Damage assessment in ANCA-associated vasculitis. Curr Rheumatol Rep. 2012;14:494-500.

6. Han CW, Lee EJ, Iwaya T, Kataoka H, Kohzuki M. Development of the Korean version of Short-Form 36-Item Health Survey: health related QOL of healthy elderly people and elderly patients in Korea. Tohoku J Exp Med. 2004;203: 189-94.

7. Cruikshank W, Center DM. Modulation of lymphocyte migration by human lymphokines. II. Purification of a lymphotactic factor (LCF). J Immunol. 1982; 128:2569-74

8. Cruikshank WW, Kornfeld H, Center DM. Interleukin-16. J Leukoc Biol. 2000; 67:757-66.

9. Wilson KC, Center DM, Cruikshank WW. The effect of interleukin-16 and its precursor on T lymphocyte activation and growth. Growth Factors. 2004;22: 97-104.

10. Lee S, Kaneko H, Sekigawa I, Tokano Y, Takasaki Y, Hashimoto H. Circulating interleukin-16 in systemic lupus erythematosus. Br J Rheumatol. 1998;37: 1334-7.

11. Cho ML, Jung YO, Kim KW, Park MK, Oh HJ, Ju JH, et al. IL-17 induces the production of IL-16 in rheumatoid arthritis. Exp Mol Med. 2008;40:237-45.

12. Murota A, Suzuki K, Kassai Y, Miyazaki T, Morita R, Kondo Y, et al. Serum proteomic analysis identifies interleukin 16 as a biomarker for clinical response during early treatment of rheumatoid arthritis. Cytokine. 2016;78: 87-93.

13. Klimiuk PA, Goronzy JJ, Weyand CM. IL-16 as an anti-inflammatory cytokine in rheumatoid synovitis. J Immunol. 1999;162:4293-9. 
14. Jennette JC, Falk RJ, Hu P, Xiao H. Pathogenesis of antineutrophil cytoplasmic autoantibody-associated small-vessel vasculitis. Annu Rev Pathol. 2013;8:139-60.

15. Kawabata K, Makino T, Makino K, Kajihara I, Fukushima S, Ihn H. IL-16 expression is increased in the skin and sera of patients with systemic sclerosis. Rheumatology (Oxford). 2020;59:519-523.

16. Glass WG, Argentieri RL, Bracht M, Farrell FX, Das AM, Del Vecchio AM, et al. Generation of bleomycin-induced lung fibrosis is independent of IL-16. Cytokine. 2009;46:17-23.

17. Roth S, Solbach W, Laskay T. IL-16 and MIF: messengers beyond neutrophil cell death. Cell Death Dis. 2016;7:e2049.

18. Cruikshank WW, Kornfeld H, Center DM. Signaling and functional properties of interleukin-16. Int Rev Immunol. 1998;16:523-40.

\section{Publisher's Note}

Springer Nature remains neutral with regard to jurisdictional claims in published maps and institutional affiliations.

Ready to submit your research? Choose BMC and benefit from:

- fast, convenient online submission

- thorough peer review by experienced researchers in your field

- rapid publication on acceptance

- support for research data, including large and complex data types

- gold Open Access which fosters wider collaboration and increased citations

- maximum visibility for your research: over $100 \mathrm{M}$ website views per year

At BMC, research is always in progress.

Learn more biomedcentral.com/submissions 